\title{
Genetic variant predicts bevacizumab- induced hypertension in ECOG-5103 and ECOG-2100
}

B P Schneider ${ }^{*} 1,17, \mathrm{~L} \mathrm{Li}^{2,17}$, F Shen ${ }^{1}, \mathrm{~K}$ D Miller ${ }^{1}, \mathrm{M} \mathrm{Radovich}^{3}, \mathrm{~A}^{\prime}$ Neill $^{4}$, R J Gray ${ }^{4}$, D Lane ${ }^{1}$, D A Flockhart ${ }^{5}$, G Jiang ${ }^{2}$, Z Wang ${ }^{2}$, D Lai ${ }^{2}$, D Koller ${ }^{2}$, J H Pratt ${ }^{1}$, C T Dang ${ }^{6}$, D Northfelt ${ }^{7}$, E A Perez ${ }^{8}$, T Shenkier ${ }^{9}$, M Cobleigh ${ }^{10}, \mathrm{M} \mathrm{L}$ Smith $^{11}$, E Railey ${ }^{11}$, A Partridge ${ }^{12}$, J Gralow ${ }^{13}$, J Sparano ${ }^{14}$, N E Davidson ${ }^{15}$, T Foroud $^{2,18}$ and G W Sledge ${ }^{16,18}$

\begin{abstract}
${ }^{1}$ Department of Medicine, Indiana University School of Medicine, Indianapolis, IN 46202, USA; ${ }^{2}$ Department of Medical \& Molecular Genetics, Indiana University School of Medicine, Indianapolis, IN, 46202, USA; ${ }^{3}$ Department of General Surgery, Indiana University School of Medicine, Indianapolis, IN 46202, USA; ${ }^{4}$ Department of Biostatistics and Computational Biology, Dana Farber Cancer Institute, Boston, MA 02215, USA; ${ }^{5}$ Indiana Institute for Personalized Medicine, Indiana University School of Medicine, Indianapolis, IN 46202, USA; ${ }^{6}$ Department of Medicine, Memorial Sloan Kettering Cancer Center, New York, NY 10065, USA; ${ }^{7}$ Department of Medicine, Mayo Clinic, Scottsdale, AZ 85054, USA; ${ }^{8}$ Mayo Clinic, Jacksonville, FL 32224, USA; ${ }^{9}$ BCCA - Vancouver Cancer Center, Vancouver, BC, V5Z 4E6, USA; ${ }^{10}$ Department of Internal Medicine, Rush-Presbyterian-St. Luke's Medical Center, Chicago, IL 60612, USA; ${ }^{11}$ Research Advocacy Network, Plano, TX 75093, USA; ${ }^{12}$ Department of Medical Oncology, Dana Farber Cancer Institute, Boston, MA 02215, USA; ${ }^{13}$ University of Washington, Seattle, WA 98195, USA; ${ }^{14}$ Department of Oncology, Montefiore Hospital and Medical Center, Bronx, NY 10467, USA; ${ }^{15}$ Cancer Institute and University of Pittsburgh Cancer Center, Pittsburgh, PA 15232, USA and ${ }^{16}$ Department of Medicine, Stanford University, Stanford, CA 94305, USA
\end{abstract}

Background: Bevacizumab has broad anti-tumour activity, but substantial risk of hypertension. No reliable markers are available for predicting bevacizumab-induced hypertension.

Methods: A genome-wide association study (GWAS) was performed in the phase III bevacizumab-based adjuvant breast cancer trial, ECOG-5103, to evaluate for an association between genotypes and hypertension. GWAS was conducted in those who had experienced systolic blood pressure (SBP) $>160 \mathrm{~mm} \mathrm{Hg}$ during therapy using binary analysis and a cumulative dose model for the total exposure of bevacizumab. Common toxicity criteria (CTC) grade 3-5 hypertension was also assessed. Candidate SNP validation was performed in the randomised phase III trial, ECOG-2100.

Results: When using the phenotype of SBP $>160 \mathrm{~mm} \mathrm{Hg}$, the most significant association in SV2C (rs6453204) approached and met genome-wide significance in the binary model $\left(P=6.0 \times 10^{-8} ; \mathrm{OR}=3.3\right)$ and in the cumulative dose model $\left(P=4.7 \times 10^{-8}\right.$; $H R=2.2$, respectively. Similar associations with rs6453204 were seen for CTC grade 3-5 hypertension but did not meet genomewide significance. Validation study from ECOG-2100 demonstrated a statistically significant association between this SNP and grade $3 / 4$ hypertension using the binary model $(P$-value $=0.037 ; \mathrm{OR}=2.4)$.

Conclusions: A genetic variant in SV2C predicted clinically relevant bevacizumab-induced hypertension in two independent, randomised phase III trials.

\footnotetext{
*Correspondence: Dr BP Schneider; E-mail: bpschnei@iupui.edu

${ }^{17}$ These authors contributed equally to this work.

${ }^{18}$ These authors contributed equally to this work.
}

Received 2 May 2014; revised 26 June 2014; accepted 8 July 2014; published online 12 August 2014

(c) 2014 Cancer Research UK. All rights reserved 0007 -0920/14 
Bevacizumab is a monoclonal antibody that targets vascular endothelial growth factor (VEGFA). Although this drug has broad anti-tumour properties, there are no validated biomarkers to predict which subgroup of patients will respond or experience toxicity. The inability to enrich for the optimal population has been a barrier to maximum success. To date, bevacizumab has demonstrated clinically meaningful activity in colon cancer (Hurwitz et al, 2004), lung cancer (Sandler et al, 2006), renal-cell cancer (Yang et al, 2003), cervical cancer (Tewari et al, 2013) and glioblastoma multiforme (Walter Taal et al, 2013). Bevacizumab also gained accelerated US-FDA approval for metastatic breast cancer based, in part, on the results of ECOG-2100 (Miller et al, 2007), a randomised phase III trial of paclitaxel with or without bevacizumab. On the basis of less absolute differences in progression-free survival in later trials (AVADO, RIBBON-I, and RIBBON-II (Miles et al, 2010; Brufsky et al, 2011; Robert et al, 2011)), no overall survival benefit, and due to the unique and unpredictable toxicity profile, bevacizumab's US-FDA approval for breast cancer was rescinded (FDA NEWS RELEASE, 2011). Despite this, bevacizumab has remained an approved treatment for breast cancer in the European Union. The early success of bevacizumab in the metastatic setting led to the development of ECOG-5103, which tested a standard backbone of chemotherapy with concurrent or concurrent plus sequential bevacizumab in the adjuvant setting with results pending maturation of efficacy data. Two other phase-III adjuvant breast cancer trials, BETH and BEATRICE (Cameron et al, 2013; Slamon et al, 2013), have been negative, highlighting the limitations of bevacizumab for breast cancer in the absence of a successful efficacy marker.

A major limitation of bevacizumab across all cancer types has been the inability to predict $a$ priori which patients are most likely to obtain substantial benefit or toxicity from treatment (Schneider and Sledge, 2011). Validated biomarkers to predict either the efficacy or toxicity of bevacizumab are lacking, although germline DNA variants have been previously nominated (Schneider et al, 2012). Hypertension is the most common, serious bevacizumabinduced toxicity, which can occasionally be life threatening. Herein we have identified and validated a genetic biomarker for bevacizumab-induced hypertension in the adjuvant breast cancer trial, ECOG-5103 (whose efficacy data were not yet available for this analysis) and the metastatic breast cancer trial, ECOG-2100.

\section{MATERIALS AND METHODS}

Genome-wide association study for SNP discovery in ECOG-5103

ECOG-5103 overview. ECOG-5103 (Figure 1) was a phase-III adjuvant breast cancer trial that randomised 4994 patients to

A

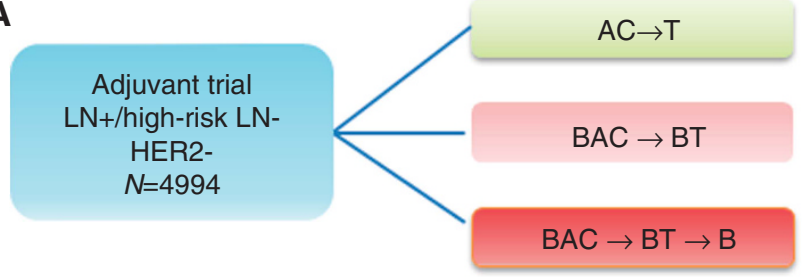

B

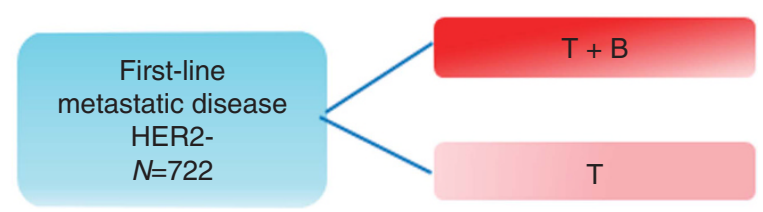

A=doxorubicin; $\mathrm{C}=$ cyclophosphamide; $\mathrm{B}=$ bevacizumab; $\mathrm{T}=$ paclitaxel; $\mathrm{LN}=$ lymph node

Figure 1. Trial schemas for (A) ECOG-5103 and (B) ECOG-2100. doxorubicin and cyclophosphamide for four cycles, followed by 12 weeks (four cycles) of weekly paclitaxel ( $\operatorname{arm~A)~or~to~the~same~}$ chemotherapy with either concurrent bevacizumab ( $\operatorname{arm} B)$ or concurrent plus sequential bevacizumab every 3 weeks (10 additional cycles) (arm C). Germline DNA was available from 4033 patients (Figure 2). For those patients that received bevacizumab (arms B and $\mathrm{C}$ ), analyses were performed to identify SNP genotypes that were associated with hypertension. This correlative study was approved by the Indiana University Institutional Review Board.

ECOG-5103 genotyping, quality control (QC), race designation, and imputation. Owing to the availability of germline DNA, genotyping was performed at two independent time points in non-overlapping study subsets. DNA from an initial 2209 patients was genotyped by Illumina Genotyping Services (San Diego, CA, USA) using the HumanOmnil-Quad ( $>1$ million SNPs) array. An additional 1222 patients were also genotyped by Illumina Genotyping Services using the Human OmniExpress (741 000 SNPs) array. Both sample sets used the Illumina BeadChip microarray platform for genotyping and the Illumina GenomeStudio software for initial genotyping calls. Of note, SNPs on the OmniExpress were a subset of those on the HumanOmnil-Quad. Those SNPs not on the HumanOmni1-Quad were obtained through imputation in the second sample. Genotypes from both sample sets were imputed to the level of the 1000 genome project. Imputation was performed with the 1000 Genomes Phase-Iintegrated variant set as reference using the IMPUTE2 software, and all SNPs were mapped to the human genome version GRCh37.3. Prior to imputation, SNPs with missing rate $>5 \%$, Hardy-Weinberg Equilibrium (HWE) $P$-values $<0.0001$, or minor allele frequency (MAF) $<3 \%$ were excluded. A principal component analysis was performed using Eigenstrat (Price et al, 2006) and data from 11 HapMap phase-III populations to identify clusters using the first two eigenvectors computed using all SNPs (Supplementary Figure S1). Only samples clustering with the European American reference set were retained.

ECOG-5103 case and control definitions for bevacizumabinduced hypertension

Cases: Blood pressure values were collected as part of standard clinical assessment prior to administration of therapy throughout the conduct of the trial. The goal for phenotype development was to combine clinically relevant and established parameters with the highest severity that would still allow reasonable statistical power to detect an association (Supplementary Information 1). Consistent with the definition for stage-II hypertension developed by the Joint National Committee (JNC 7) (http://www.nhlbi.nih.gov/guidelines/ hypertension/express.pdf), first occurrence of a systolic blood pressure (SBP) $>160 \mathrm{~mm} \mathrm{Hg}$ was selected to define a case. Of note, a baseline $\mathrm{SBP}>160 \mathrm{~mm} \mathrm{Hg}$ was also an exclusion criterion for eligibility to enrolment in the parent trial. In addition, another analysis classified those individuals with grade $3-5$ hypertension as cases, determined by the common toxicity criteria (CTC) version 3.0 (CTC v. 3.0).

Controls: Controls included all patients in arm B and arm $\mathrm{C}$ who met all the following criteria: (1). SBP $<140 \mathrm{~mm} \mathrm{Hg}$ at baseline without use of an anti-hypertensive; (2) maintained a median $\mathrm{SBP}<140 \mathrm{~mm} \mathrm{Hg}$ throughout therapy; (3) did not meet any of the definitions for cases as outlined above; and (4) received all prescribed doses of bevacizumab with follow-up at least 3 months beyond the last dose. Specifically, patients that had bevacizumab held or modified for other reasons (i.e., disease progression or other toxicity) were excluded from control designation. See consort diagram; Figure 2.

Statistical analysis. Two hypotheses were evaluated in the statistical analyses. First, a standard case-control association (binary) analysis was performed to identify SNPs associated with the presence or absence of hypertension. Second, an analysis was performed to identify SNPs associated with the cumulative bevacizumab dose before becoming a case (i.e., cumulative dose 
A
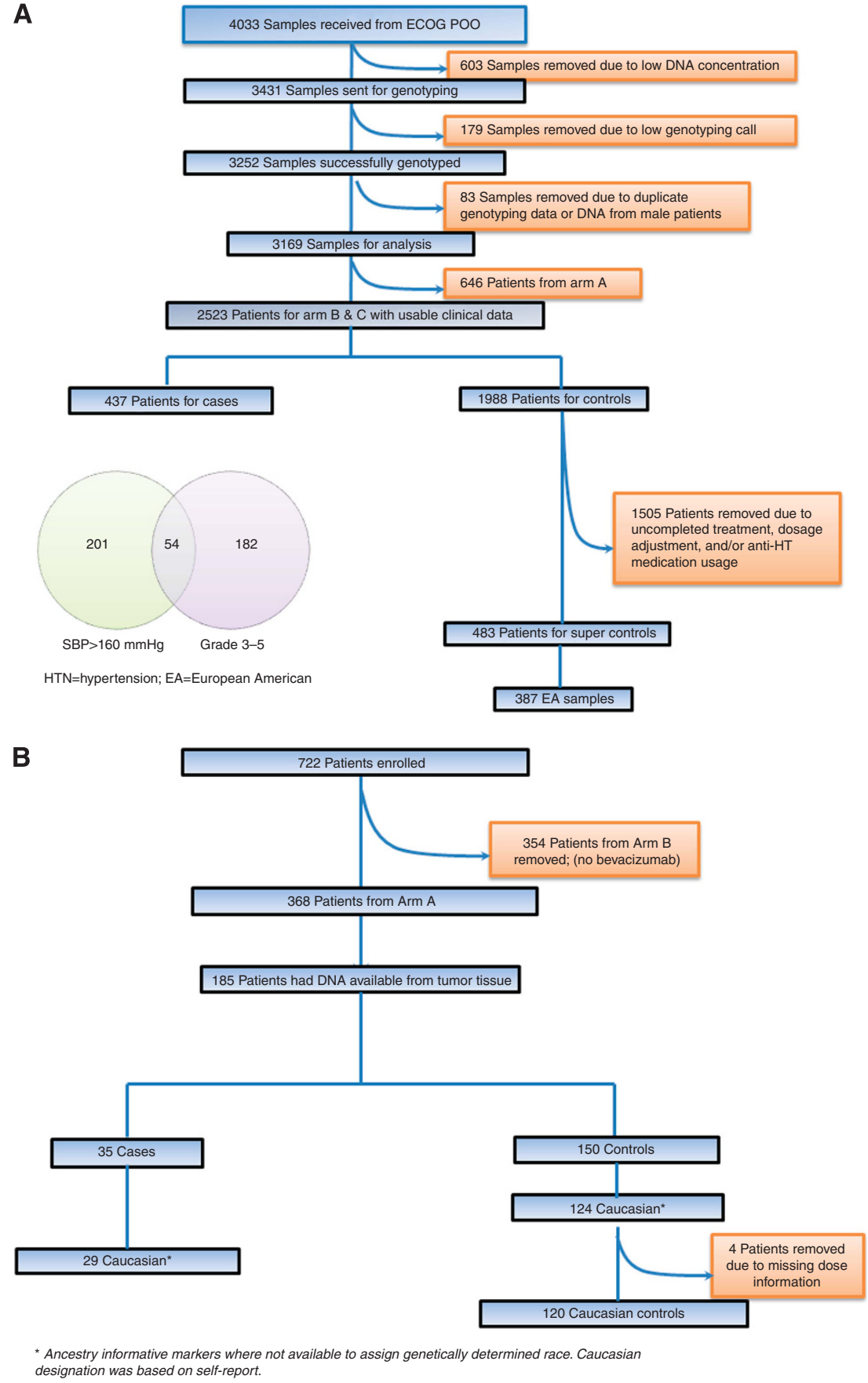

Figure 2. Consort diagram for (A) ECOG-5103 and (B) ECOG-2100.

model). The cumulative dose model was implemented with a Cox proportional hazard model, in which the dose was computed as the full dose or censored. Age ( $\leqslant 50 v s>50)$ and obesity (body mass index (BMI) > 30; yes/no) were considered as covariates in both analyses. The $P$-value threshold was set at $P$-value $<0.05$ for inclusion of covariates in the regression model.

SNPs available on the HumanOmnil-Quad array were used as the common basis for all statistical analysis. An additive model of SNP effect was used when testing all hypotheses. The case-control analysis was conducted using SNPTEST v2.4 (https://mathgen. stats.ox.ac.uk/genetics_software/snptest/snptest.html). The cumulative dose model was conducted in $\mathrm{R}$ (www.r-project.org) using the coxph function. For all analyses, the $P$-value threshold for genome-wide significance was set at $5 \times 10^{-8}$ to correct for multiple comparisons (Barsh et al, 2012).

\section{Candidate SNP validation in ECOG-2100}

ECOG-2100 overview. ECOG-2100 was a phase-III breast cancer trial that randomised patients with metastatic breast cancer that had not previously received chemotherapy in the metastatic setting to either paclitaxel ( $\operatorname{arm} B$ ) or paclitaxel with bevacizumab ( $\operatorname{arm~A)}$; Figure 1. The results of ECOG-2100 have been previously reported (Miller et al, 2007). 367 patients had DNA available from formalinfixed paraffin-embedded (FFPE) primary tumours.

Case and control designation from ECOG-2100: Only patients randomised to arm A with DNA available for genotyping were considered ( $n=185$ subjects). Raw blood pressure values were not available for ECOG-2100; therefore, grade 3-5 hypertension as determined by CTC version 2.0 criteria was used (compared with 
version 3.0 for ECOG-5103) as the only phenotype definition. Grade 4 hypertension by CTC version 2.0 criteria was similar to version 3.0 (e.g., hypertensive crisis for both). Grade-3 hypertension for version 2.0 was defined as requiring therapy or more intensive therapy than previously, whereas for version 3.0 it was defined as requiring $>1$ drug or more intensive therapy than previously. Controls were those patients that did not have CTC version 2.0 grade 3-5 hypertension at any point throughout treatment.

ECOG-2100 genotyping and statistical analysis. Germline DNA was not available from E2100, and thus DNA was extracted from 20-micron paraffin-embedded tissue sections from the primary tumour using the DNeasy Tissue kit (Qiagen, Valencia, CA, USA) (Schneider et al, 2008). The SNP in SVC2, rs6453204, was genotyped in 185 subjects. Genotyping was performed after a pre-amplification procedure using a TaqMan SNP Genotyping Assay (C_ 30765975 10 (Life Technologies, Carlsbad, CA, USA)), which was performed on the ABI 7900 Fast Real Time PCR platform (Life Technologies). Allelic discrimination was determined during a post-amplification plate read by the TaqMan Genotyper software (Life Technologies). The quality of the SNP genotyping was assessed by confirming that the SNP did not violate HWE and had MAF similar to previously reported frequencies. The same analytic models used in the ECOG5103 analyses were employed in the ECOG-2100 statistical analysis. Only age was considered as a covariate in the regression model as BMI information was not available. The $P$-value threshold for the SNP and covariate selection was 0.05 .

\section{RESULTS}

Clinical associations with hypertension in ECOG-5103. The percentage of patients who had a hypertensive event defined as $\mathrm{SBP}>160 \mathrm{~mm} \mathrm{Hg}$ and CTC grade 3-5 hypertension were $10.4 \%$ and $9.5 \%$, respectively. The median first hypertensive event occurred at cycle \#10 for the SBP $>160 \mathrm{~mm} \mathrm{Hg}$ phenotype and at cycle \#11 for CTC grade 3-5 hypertension phenotype, respectively. Race was significantly associated with hypertension. AfricanAmericans were 1.63 -fold more likely to develop hypertension during treatment with bevacizumab as compared with individuals of other races $(P=0.0039)$. To obviate bias, the analysis sample was limited to individuals of European descent. Age $>50$ years $\left(P=4.3 \times 10^{-7}\right)$ and obesity $\left(P=3.7 \times 10^{-4}\right)$ were correlated with an increased likelihood of hypertension. Both age and obesity were included as covariates in analyses.

Statistical association results in ECOG-5103. The sample sizes and number of SNPs available for each phenotype are provided in Table 1. When using SBP $>160 \mathrm{~mm} \mathrm{Hg}$ as the hypertension criterion, the most significant association of a genetic variant with bevacizumab-induced hypertension was rs6453204, a SNP in the SV2C gene located on chromosome 5 with a MAF of 7\%. The association approached genome-wide significance in the binary model $(P=6.0$ $\left.\times 10^{-8} ; \mathrm{OR}=3.3\right)$ and met genome-wide significance in the cumulative dose model $\left(P=4.7 \times 10^{-8} ; \mathrm{HR}=2.2\right)$; Figure $3 \mathrm{~A}$ and $\mathrm{B}$ and Figure 4. See Supplementary Information 2 for comprehensive association results. No SNPs provided genome-wide evidence of association using both binary and cumulative dose model, in which cases were defined as those meeting grade $3 / 4$ hypertension. The $P$-values for association with the SV2C SNP, rs6453204 were $6.4 \times 10^{-5}(\mathrm{OR}=2.0)$ and $3.0 \times 10^{-4}(\mathrm{HR}=2.2)$ using the binary model and the cumulative dose model, respectively.

Validation of SV2C in ECOG-2100. We then performed an independent validation of the SV2C SNP in the clinical trial ECOG2100. As previously reported, $14.8 \%$ of patients experienced CTC grade 3-4 hypertension in E2100 (Miller et al, 2007). The median first occurrence of CTC grade 3-4 hypertension in the subset of 185 patients who had genotyping data appeared at cycle \#1.5 but ranged from cycle \#1-29. We found a statistically significant association of rs6453204 with bevacizumab-induced grade 3-5 hypertension using the binary model $(P=0.037 ; \mathrm{OR}=2.4)$. Similar results were obtained using the cumulative dose model, but did not reach statistical significance $(P=0.091 ; \mathrm{HR}=1.9)$; Table 2 .

\section{DISCUSSION}

This study revealed a compelling association between bevacizumab-induced hypertension and an SNP in SV2C (rs6453204) in two randomised phase III trials. Evidence of association was initially found in the clinical trial ECOG-5103 and further validated in ECOG-2100. This represents one of the first pharmacogenomic studies to uncover a predictive biomarker for hypertension using a genome-wide approach within a phase-III breast cancer trial and candidate validation in another phase III trial. Although ECOG-5103 and ECOG-2100 were trials designed to test bevacizumab for breast cancer, it is highly likely that the toxicities and corresponding predictive biomarkers transcend tumour type or setting, as the tumour has little bearing on druginduced toxicity. The association with rs6453204 in ECOG-5103 was found using both a binary and cumulative dose model. The former simply classified each patient as a case or control, whereas the later model accounted for the total drug exposure and was a successful methodology previously employed by Baldwin et al (2012). Thus, we have observed consistent results using both a dichotomous and a quantitative phenotype.

Synaptic vesicle protein 2 (SV2) is encoded by a small gene family with three established isoforms: SV2A, SV2B, and SV2C (Janz and Sudhof, 1999). The SV2C gene encodes for the SV2C protein, and rs6453204 is an intronic SNP. Recent studies have identified SNPs in SV2C, which are associated with a gene-smoking interaction on risk of Parkinson's disease (Hill-Burns et al, 2013) and the response to atypical antipsychotics (Ramsey et al, 2013). In addition, an SNP close to $S V 2 C$ has been reported as new candidate loci for impact on venous thromboembolism and coagulation pathways (Tang et al, 2013). These studies suggest that SV2C may have various biological and pharmacological implications. SV2 proteins also appear to be specific to both neurons and endocrine cells, and function to maintain pools of vesicles for calciuminduced exocytosis (Janz et al, 1999). Specifically relevant to this phenotype, SV2C has a role in release of catecholamines from

Table 1. Sample and SNP information for hypertension phenotypes in ECOG-5103

\begin{tabular}{|l|c|c|c|c|c|}
\hline Phenotype definition & Cases $(\boldsymbol{n})$ & Controls $(\boldsymbol{n})$ & SNPs with INFO $<\mathbf{0 . 3}^{\text {a }}$ & SNPs with MAF $<\mathbf{0 . 0 3}$ & Total SNPs used \\
\hline Systolic blood pressure $>160 \mathrm{~mm} \mathrm{Hg}$ & 195 & 387 & 76296 & 114761 & 810372 \\
\hline Grade 3/4 hypertension & 177 & 387 & 76296 & 114847 & 810286 \\
\hline a This is reported by IMPUTE2 as a measurement of imputation quality. Lower value indicates higher uncertainty of imputation. \\
\hline
\end{tabular}



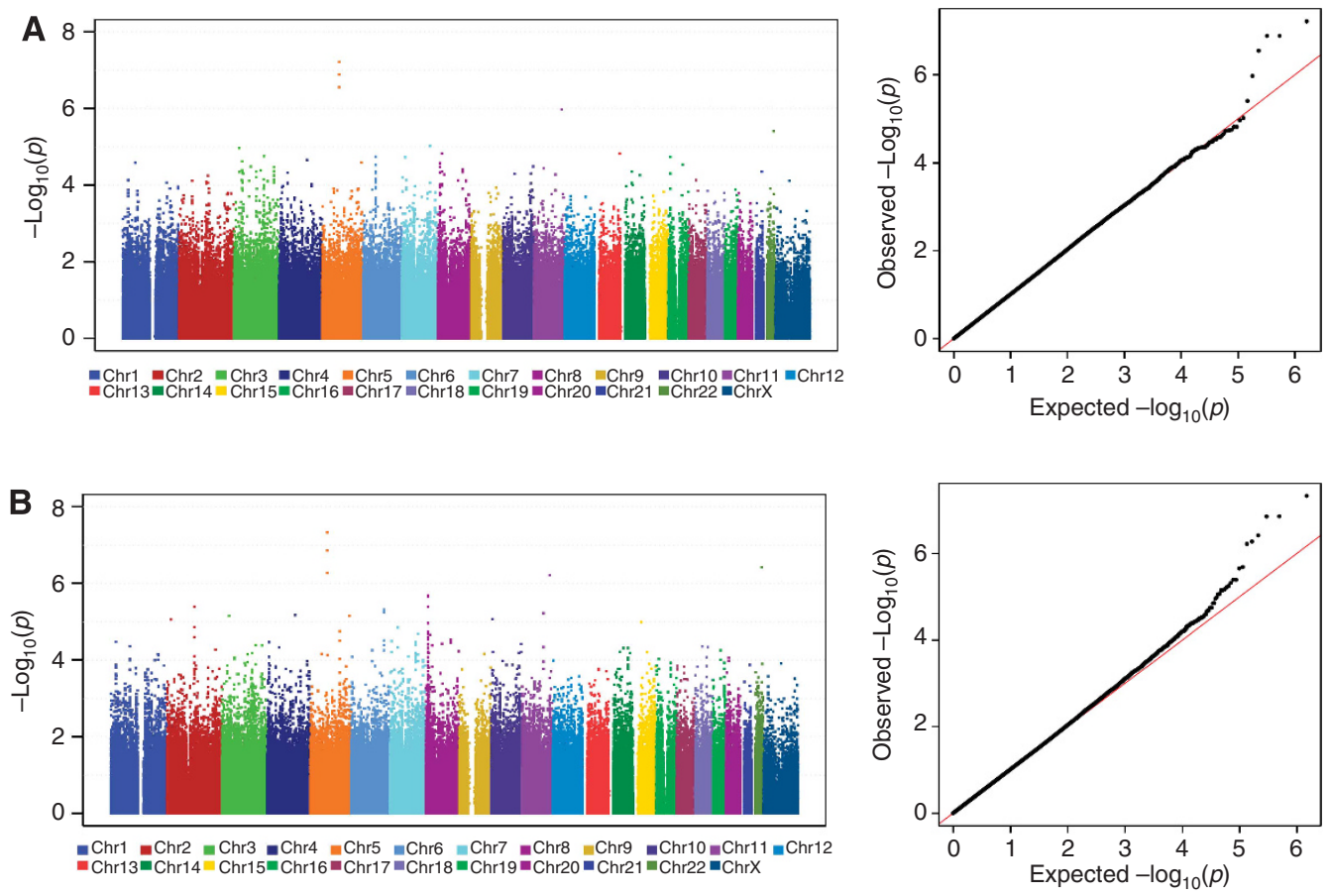

Figure 3. Manhattan plot (left) and quantile-quantile plot (right) of genome-wide association results in E5103 under different models of hypertension. X-axis indicates the chromosomal position of each SNP analysed; Y-axis denotes magnitude of the evidence for association, shown as $-\log _{10}(P$-value). (A). Binary model for systolic blood pressure $>160 \mathrm{~mm} \mathrm{Hg}$. (B). Cumulative dose model for the systolic blood pressure $>160 \mathrm{~mm} \mathrm{Hg}$.

adrenal chromaffin cells (Xu and Bajjalieh, 2001), which certainly might represent a potential mechanism for impact on blood pressure. Production of the sodium-retaining hormone aldosterone may be modulated through adrenocortical connections with the adrenal medulla (Gallo-Payet et al, 1987). Additional functional work is needed to elucidate mechanism, but more importantly, to shed insight to novel therapeutics.

A strength of this study was the detailed review of the clinical data in ECOG-5103 and ECOG-2100. All of the patients in the discovery set from ECOG-5103 were part of the same clinical trial with rigorous attention to consistent treatment and collection of clinical data. Similarly, the validation cohort was also part of a clinical trial, ECOG-2100, that provided similar rigorous patientto-patient consistency with regard to treatment protocol and collection of data. Unlike the evaluation of random patients who received bevacizumab, the use of patients from a clinical trial eliminates many innate confounders. Cases and controls were carefully adjudicated based on stringent phenotype criteria, and the companion genotyping data underwent substantial QC and filtering. The control samples were carefully evaluated and patients having any evidence of prior hypertension or who did not complete a substantial course of bevacizumab were removed from the analysis. We believe that this careful phenotypic review provided the best opportunity for identification of a true genetic association.

One of the challenges of detecting associations with druginduced hypertension is the dynamic and multifactorial nature of hypertension and the variability in measurement. We chose several complementary approaches to define the phenotype of druginduced hypertension. Each was selected because it provided additional insight. For the primary analysis, we selected stage II hypertension determined by the Joint National Committee (JNC-7) as a value $(\mathrm{SBP}>160 \mathrm{~mm} \mathrm{Hg})$ that had both clinical relevance and was sufficiently frequent in this population to allow for statistical power to detect an association. This was the same definition used to exclude patients from entry in the parent trial owing to uncontrolled baseline hypertension. We recognised there were inherent limitations to the use of a single blood pressure value obtained in an outpatient clinic. Nonetheless, for a pharmacogenomics study of this size, this represented the most objective, standardised measure readily available. Further, this represented the same data that are routinely used in the community setting to make decisions as to whether to add an anti-hypertensive or to stop the drug. The other phenotype, CTC grade 3-5 hypertension, was commonly collected, but was subjective and based on the threshold for an investigator to either add an anti-hypertensive or to consider the hypertensive event 'life threatening'. Specifically, many investigators were aware of this drug-specific toxicity based on their prior experience, and may have been tempted to treat with anti-hypertensive agents early to prevent problems later. Thus, although the threshold of a SBP $>160 \mathrm{~mm} \mathrm{Hg}$ may not represent the optimal value, we believe an absolute threshold was the proper approach for this phenotype.

Prior data have suggested that those patients who develop hypertension may fare better from an efficacy standpoint (Olsen et al, 2004; Schneider et al, 2008; Jain et al, 2010; Rini et al, 2011), although there are conflicting reports (Hurwitz et al, 2010). This type of analysis was complicated by the non-uniformity of the phenotype (as discussed above) and the heterogeneous benefit for bevacizumab across tumour types and settings. If indeed, those who get hypertension are those destined to benefit most, continuation of therapy despite toxicity is critical. Even if an association between efficacy and toxicity is not valid, however, optimal management of these patients remains an important goal. For these reasons, a biomarker that can predict who might be at risk for this toxicity might be highly valuable in the clinical setting. For those deemed to be at increased risk of significant hypertension, close monitoring with early intervention or use of prophylactic anti-hypertensive therapies could be considered. Additional information that can be gleaned from a genetic 
biomarker is the potential to understand the underlying biology of the toxicity. Fundamental biological insights can further lead to novel therapeutic development for treatment or prevention. If

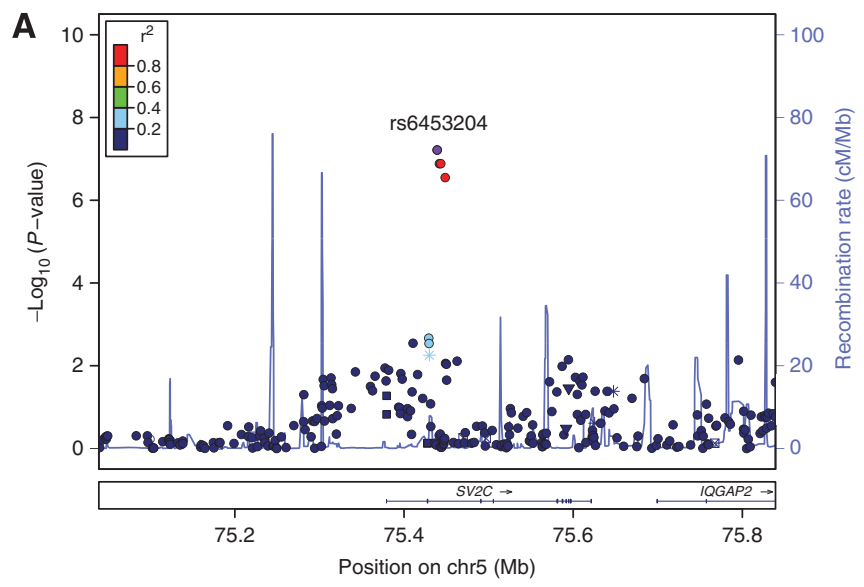

B

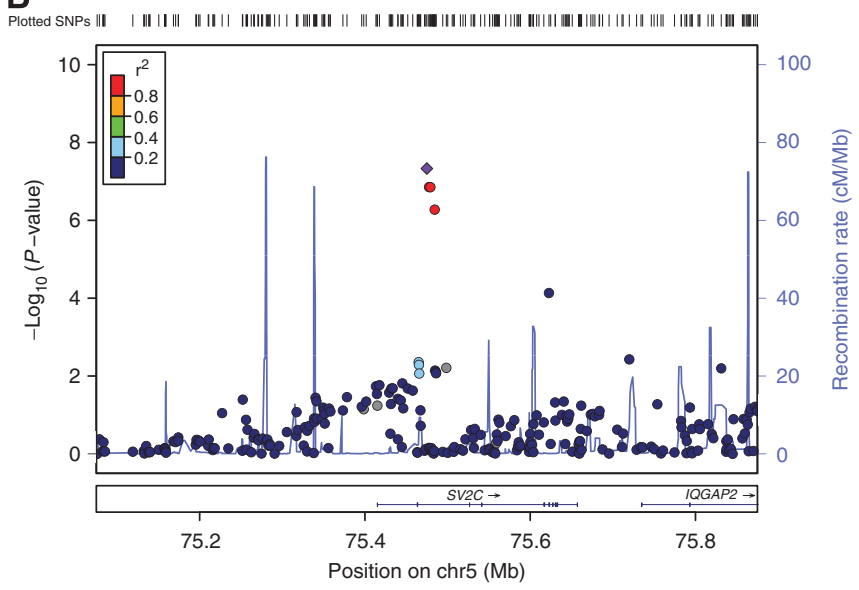

Figure 4. Association results in the SVC2 region for models of hypertension defined as systolic blood pressure $>160 \mathrm{~mm} \mathrm{Hg}$. Results for all genotyped and imputed SNPs that passed quality-control parameters are shown. $\mathrm{X}$-axis denotes position of the SNP in the region on chromosome $5 ; \mathrm{Y}$-axis denotes magnitude of the evidence for association, shown as $-\log _{10}(P$-value). The most significantly associated SNP is denoted with a purple diamond. The extent of linkage disequilibrium (as measured by $r^{2}$ ) between each SNP and the most significantly associated SNP is indicated by the colour scale at top left. Larger values of $r^{2}$ indicate greater linkage disequilibrium with the most significant SNP. (A) Binary model. (B) Cumulative dose model. indeed there is a link between hypertension and effectiveness of the drug, biomarkers for toxicity may also serve as markers for efficacy. More broadly, an understanding of bevacizumab-induced hypertension might shed light on the pathophysiology and treatments for common disorders such as essential hypertension.

Prior studies have evaluated an association between candidate SNPs and bevacizumab-induced hypertension. We previously identified an association between SNPs in VEGFA and protection from grade 3/4 hypertension (Schneider et al, 2008) A similar finding was demonstrated with sunitinib (Kim et al, 2012). In this study, we specifically probed the genotyped SNPs in VEGFA (rs2010963, rs1570360) that had previously been associated with hypertension induced by an anti-angiogenic agent, and none demonstrated a statistically significant association in E5103 regardless of phenotype. Thus, although these prior results were provocative, they could not be validated in this much larger clinical trial.

A weakness of this study is the limited, phenotypic information available from ECOG-2100; specifically only CTC version 2.0 grade $3 / 4$ hypertension could be analysed. Although similar, it should be pointed out that the criterion for hypertension was slightly different between version 2.0 and version 3.0, the latter of which was used in ECOG-5103. Therefore, it is likely that the association with the SNP in SV2C may have been underestimated in the validation cohort owing to a suboptimal phenotype definition. In addition, it is possible that the characteristics and the performance status of patients in the metastatic setting (ECOG2100) may not mirror those of the patients in the adjuvant setting (ECOG-5103), which might subsequently impact the hypertension phenotype. Unfortunately, germline DNA was not available in ECOG-2100, and FFPE tumour-derived DNA was used. Therefore, somatic mutations or loss of heterozygosity could have reduced the power to replicate the SNP in SV2C association with drug-induced hypertension. This concern was mitigated as there was no deviation from HWE, and the MAF was similar to what was expected in the general population. We also examined data for $S V 2 C$ in the Cancer Genome Atlas and found no evidence of recurrent mutation, loss of heterozygosity, nor recurrent copynumber aberrations (Cerami et al, 2012; Gao et al, 2013). Another limitation was the insufficient number of cases and controls to identify markers for patients of African descent. Data from ECOG5103 would support that African-Americans are more likely to experience this toxicity. Thus, work directed at identifying a marker for the group most likely to experience this toxicity is of utmost importance.

In summary, we have identified an SNP in SV2C that predicts bevacizumab-induced hypertension. Future studies testing early clinical intervention as well as elucidation of the functionality of this SNP are warranted and are ongoing. Also, evaluation of $S V 2 C$ (as well as other candidates) as a marker for efficacy will be conducted in the E5103 trial, as efficacy data become available.

Table 2. Summary of $P$-values and OR by phenotype and analysis model for rs6453204

\begin{tabular}{|c|c|c|c|c|c|c|}
\hline Phenotype & Model & Analysis & $P$-value E5103a & $P$-value E2100 & OR/HR E5103 & OR/HR E2100 \\
\hline \multirow{2}{*}{$\mathrm{SBP}>160$} & Binary & Additive & $6.0 \times 10^{-8}$ & NA & 3.3 & NA \\
\hline & Cumulative dose & Additive & $4.7 \times 10^{-8}$ & NA & 2.2 & NA \\
\hline \multirow[b]{2}{*}{ Grade 3/4 } & Binary & Additive & $3.0 \times 10^{-4}$ & 0.037 & 2.2 & 2.4 \\
\hline & Cumulative dose & Additive & $6.4 \times 10^{-5}$ & 0.091 & 2.0 & 1.9 \\
\hline \multicolumn{7}{|c|}{$\begin{array}{l}\text { Abbreviations: } \mathrm{HR}=\text { hazard ratio; } \mathrm{NA}=\text { not available; } \mathrm{OR}=\text { odds ratio; } \mathrm{SBP}=\text { systolic blood pressure. } \\
\mathrm{a}_{\text {S }} \text { tatistical significance is defined as } P \text {-value }<5.0 \times 10^{-8} \text {. } \\
\mathrm{b}_{\text {Statistical significance is defined as } P \text {-value }<0.05}\end{array}$} \\
\hline
\end{tabular}




\section{ACKNOWLEDGEMENTS}

This study was coordinated by the Eastern Cooperative Oncology Group (Robert L Comis) and supported in part by Public Health Service Grants CA23318, CA66636, CA21115, CA49883, CA14958, CA16116, CA39229, CA25224, CA12027, CA32102, CA20319, CA77202, and from the National Cancer Institute, National Institutes of Health and the Department of Health and Human Services. Biospecimens were provided by the ECOG Pathology Coordinating Office and Reference Laboratory, and the procurement and preparation of samples was supported by the Breast Cancer Research Foundation (Daniel Hayes; PI). The science was also supported by a Susan G Komen for the Cure Promise Award (BPS; PI) and CA155311-01A1 (BPS; PI).

\section{CONFLICT OF INTEREST}

The following authors have received research funds from Genentech/Roach: KDM, JG, CTD, MLS and ER; from Novatis and Amgen: JG, MLS and ER; and from Lilly, Celgene, Morphotek and Sanofi: MLS and ER. BPS is on the advisory board of Genentech (compensated). KDM has received honoraria from Genentech. JS has received consulting fees from Genentech. DAF serves on the scientific education board of the Coriell Institute and Quest Diagnostics and is an active consultant for Clinical Equilibrium Consulting LLC. The remaining authors declare no conflict of interest.

\section{DISCLAIMER}

This content is solely the responsibility of the authors and does not necessarily represent the official views of the National Cancer Institute.

\section{REFERENCES}

Baldwin RM, Owzar K, Zembutsu H, Chhibber A, Kubo M, Jiang C, Watson D, Eclov RJ, Mefford J, McLeod HL, Friedman PN, Hudis CA, Winer EP, Jorgenson EM, Witte JS, Shulman LN, Nakamura Y, Ratain MJ, Kroetz DL (2012) A genome-wide association study identifies novel loci for paclitaxelinduced sensory peripheral neuropathy in CALGB 40101. Clin Cancer Res 18(18): 5099-5109.

Barsh GS, Copenhaver GP, Gibson G, Williams SM (2012) Guidelines for genome-wide association studies. PLoS Genet 8(7): e1002812.

Brufsky AM, Hurvitz S, Perez E, Swamy R, Valero V, O’Neill V, Rugo HS (2011) RIBBON-2: a randomized, double-blind, placebo-controlled, phase III trial evaluating the efficacy and safety of bevacizumab in combination with chemotherapy for second-line treatment of human epidermal growth factor receptor 2-negative metastatic breast cancer. J Clin Oncol 29(32): 4286-4293.

Cameron D, Brown J, Dent R, Jackisch C, Mackey J, Pivot X, Steger GG, Suter TM, Toi M, Parmar M, Laeufle R, Im YH, Romieu G, Harvey V, Lipatov O, Pienkowski T, Cottu P, Chan A, Im SA, Hall PS, Bubuteishvili-Pacaud L, Henschel V, Deurloo RJ, Pallaud C, Bell R (2013) Adjuvant bevacizumabcontaining therapy in triple-negative breast cancer (BEATRICE): primary results of a randomised, phase 3 trial. Lancet Oncol 14(10): 933-942.

Cerami E, Gao J, Dogrusoz U, Gross BE, Sumer SO, Aksoy BA, Jacobsen A, Byrne CJ, Heuer ML, Larsson E, Antipin Y, Reva B, Goldberg AP, Sander C, Schultz N (2012) The cBio cancer genomics portal: an open platform for exploring multidimensional cancer genomics data. Cancer Discov 2(5): 401-404.

FDA NEWS RELEASE (2011) FDA Commissioner announces Avastin decision: Drug not shown to be safe and effective in breast cancer patients http://www.fda.gov/NewsEvents/Newsroom/PressAnnouncements/ ucm280536.htm.
Gallo-Payet N, Pothier P, Isler H (1987) On the presence of chromaffin cells in the adrenal cortex: their possible role in adrenocortical function. Biochem Cell Biol 65(6): 588-592.

Gao J, Aksoy BA, Dogrusoz U, Dresdner G, Gross B, Sumer SO, Sun Y, Jacobsen A, Sinha R, Larsson E, Cerami E, Sander C, Schultz N (2013) Integrative analysis of complex cancer genomics and clinical profiles using the cBioPortal. Sci Signal 6(269): pl1.

Hill-Burns EM, Singh N, Ganguly P, Hamza TH, Montimurro J, Kay DM, Yearout D, Sheehan P, Frodey K, McLear JA, Feany MB, Hanes SD, Wolfgang WJ, Zabetian CP, Factor SA, Payami H (2013) A genetic basis for the variable effect of smoking/nicotine on Parkinson's disease. Pharmacogenomics J 13(6): 530-537.

Hurwitz H, Douglas PS, Middleton JP, Sledge GW, Johnson DH, Reardon DA, Chen D, Rosen O (2010) Analysis of early hypertension (HTN) and clinical outcome with bevacizumab (BV). J Clin Oncol 28(suppl): 3039.

Hurwitz H, Fehrenbacher L, Novotny W, Cartwright T, Hainsworth J, Heim W, Berlin J, Baron A, Griffing S, Holmgren E, Ferrara N, Fyfe G, Rogers B, Ross R, Kabbinavar F (2004) Bevacizumab plus irinotecan, fluorouracil, and leucovorin for metastatic colorectal cancer. N Engl J Med 350(23): 2335-2342.

Jain L, Sissung TM, Danesi R, Kohn EC, Dahut WL, Kummar S, Venzon D, Liewehr D, English BC, Baum CE, Yarchoan R, Giaccone G, Venitz J, Price DK, Figg WD (2010) Hypertension and hand-foot skin reactions related to VEGFR2 genotype and improved clinical outcome following bevacizumab and sorafenib. J Exp Clin Cancer Res 29: 95.

Janz R, Goda Y, Geppert M, Missler M, Sudhof TC (1999) SV2A and SV2B function as redundant $\mathrm{Ca} 2+$ regulators in neurotransmitter release. Neuron 24(4): 1003-1016.

Janz R, Sudhof TC (1999) SV2C is a synaptic vesicle protein with an unusually restricted localization: anatomy of a synaptic vesicle protein family. Neuroscience 94(4): 1279-1290.

Kim JJ, Vaziri SA, Rini BI, Elson P, Garcia JA, Wirka R, Dreicer R, Ganapathi MK, Ganapathi R (2012) Association of VEGF and VEGFR2 single nucleotide polymorphisms with hypertension and clinical outcome in metastatic clear cell renal cell carcinoma patients treated with sunitinib. Cancer 118(7): 1946-1954.

Miles DW, Chan A, Dirix LY, Cortes J, Pivot X, Tomczak P, Delozier T, Sohn JH, Provencher L, Puglisi F, Harbeck N, Steger GG, Schneeweiss A, Wardley AM, Chlistalla A, Romieu G (2010) Phase III study of bevacizumab plus docetaxel compared with placebo plus docetaxel for the first-line treatment of human epidermal growth factor receptor 2-negative metastatic breast cancer. J Clin Oncol 28(20): 3239-3247.

Miller K, Wang M, Gralow J, Dickler M, Cobleigh M, Perez EA, Shenkier T, Cella D, Davidson NE (2007) Paclitaxel plus bevacizumab versus paclitaxel alone for metastatic breast cancer. N Engl J Med 357(26): 2666-2676.

Olsen KE, Knudsen H, Rasmussen BB, Balslev E, Knoop A, Ejlertsen B, Nielsen KV, Schonau A, Overgaard J. Danish Breast Cancer Co-operative Group (2004) Amplification of HER2 and TOP2A and deletion of TOP2A genes in breast cancer investigated by new FISH probes. Acta Oncol 43(1): $35-42$.

Price AL, Patterson NJ, Plenge RM, Weinblatt ME, Shadick NA, Reich D (2006) Principal components analysis corrects for stratification in genome-wide association studies. Nat Genet 38(8): 904-909.

Ramsey TL, Liu Q, Massey BW, Brennan MD (2013) Genotypic variation in the SV2C gene impacts response to atypical antipsychotics the CATIE study. Schizophr Res 149(1-3): 21-25.

Rini BI, Cohen DP, Lu DR, Chen I, Hariharan S, Gore ME, Figlin RA, Baum MS, Motzer RJ (2011) Hypertension as a biomarker of efficacy in patients with metastatic renal cell carcinoma treated with sunitinib. J Natl Cancer Inst 103(9): 763-773.

Robert NJ, Dieras V, Glaspy J, Brufsky AM, Bondarenko I, Lipatov ON, Perez EA, Yardley DA, Chan SY, Zhou X, Phan SC, O'Shaughnessy J (2011) RIBBON-1: randomized, double-blind, placebo-controlled, phase III trial of chemotherapy with or without bevacizumab for first-line treatment of human epidermal growth factor receptor 2-negative, locally recurrent or metastatic breast cancer. J Clin Oncol 29(10): 1252-1260.

Sandler A, Gray R, Perry MC, Brahmer J, Schiller JH, Dowlati A, Lilenbaum R, Johnson DH (2006) Paclitaxel-carboplatin alone or with bevacizumab for non-small-cell lung cancer. [Erratum appears in N Engl J Med. 2007;356(3):318] N Engl J Med 355(24): 2542-2550. 
Schneider BP, Shen F, Miller KD (2012) Pharmacogenetic biomarkers for the prediction of response to antiangiogenic treatment. Lancet Oncol 13(10): e427-e436.

Schneider BP, Sledge Jr GW (2011) Anti-vascular endothelial growth factor therapy for breast cancer: can we pick the winners? J Clin Oncol 29(18): 2444-2447.

Schneider BP, Wang M, Radovich M, Sledge GW, Badve S, Thor A, Flockhart DA, Hancock B, Davidson N, Gralow J, Dickler M, Perez EA, Cobleigh M, Shenkier T, Edgerton S, Miller KD. Ecog 2100 (2008) Association of vascular endothelial growth factor and vascular endothelial growth factor receptor-2 genetic polymorphisms with outcome in a trial of paclitaxel compared with paclitaxel plus bevacizumab in advanced breast cancer: ECOG 2100. J Clin Oncol 26(28): 4672-4678.

Slamon DJ, Swain SM, Buyse M, Martin M, Geyer CE, Im Y-H, Pienkowski T, Kim S-B, Robert NJ, Steger G, Crown J, Verma S, Eiermann W, Costantino JP, Im S-A, Mamounas EP, Schwartzberg L, Paterson A, Mackey JR, Provencher L, Press MF, Thirlwell M, Bee- Munteanu V, Henschel V, Crepelle-Flechais A, Wolmark N (2013) In Primary results from BETH, a phase 3 controlled study of adjuvant chemotherapy and trastuzumab \pm bevacizumab in patients with HER2-positive, node-positive or high risk node-negative breast cancer. San Antonio Breast Cancer Symposium. 10-14 December 2013; San Antonio, TX, USA, abstract S1-03.

Tang W, Teichert M, Chasman DI, Heit JA, Morange PE, Li G, Pankratz N, Leebeek FW, Pare G, de Andrade M, Tzourio C, Psaty BM, Basu S, Ruiter R, Rose L, Armasu SM, Lumley T, Heckbert SR, Uitterlinden AG, Lathrop M, Rice KM, Cushman M, Hofman A, Lambert JC, Glazer NL, Pankow JS, Witteman JC, Amouyel P, Bis JC, Bovill EG, Kong X,
Tracy RP, Boerwinkle E, Rotter JI, Tregouet DA, Loth DW, Stricker BH, Ridker PM, Folsom AR, Smith NL (2013) A genome-wide association study for venous thromboembolism: the extended cohorts for heart and aging research in genomic epidemiology (CHARGE) consortium. Genet Epidemiol 37(5): 512-521.

Tewari KS, Sill M, Long HJ, Ramondetta LM, Landrum LM, Oaknin A, Reid TJ, Leitao MM, Michael HE, Monk BJ (2013) Incorporation of bevacizumab in the treatment of recurrent and metastatic cervical cancer: a phase III randomized trial of the Gynecologic Oncology Group. J Clin Oncol 31(Suppl): abstract 3.

Walter Taal Oosterkamp HM, Walenkamp AME, Beerepoot LV, Hanse M, Buter J, Honkoop Boerman f, Dol A (2013) randomized phase II study of bevacizumab versus bevacizumab plus lomustine versus lomustine single agent in recurrent glioblastoma: The Dutch BELOB study. J Clin Oncol 31(Suppl): abstract 2001.

Xu T, Bajjalieh SM (2001) SV2 modulates the size of the readily releasable pool of secretory vesicles. Nat Cell Biol 3(8): 691-698.

Yang JC, Haworth L, Sherry RM, Hwu P, Schwartzentruber DJ, Topalian SL, Steinberg SM, Chen HX, Rosenberg SA (2003) A randomized trial of bevacizumab, an anti-vascular endothelial growth factor antibody, for metastatic renal cancer. $N$ Engl J Med 349(5): 427-434.

This work is published under the standard license to publish agreement. After 12 months the work will become freely available and the license terms will switch to a Creative Commons AttributionNonCommercial-Share Alike 3.0 Unported License.

Supplementary Information accompanies this paper on British Journal of Cancer website (http://www.nature.com/bjc) 